\title{
Effects of Hand and Spatial Conditions on Visual Line Bisection
}

\author{
Reiko Fukatsu, Toshikatsu Fujil, Itaru Kimura,* \\ Shun-ichi Saso* and Kyuya Kogure \\ Department of Neurology, Institute of Brain Diseases, \\ Tohoku University School of Medicine, Sendai 980, and \\ *Department of Neurology, Miyagi National Hospital, \\ Watari-gun 989-22
}

Fukatsu, R., FujiI, T., Kimura, I., Saso, S. and Kogure, K. Effects of Hand and Spatial Conditions on Visual Line Bisection. Tohoku J. Exp. Med., 1990, 161 (4), 329-333 — This study examined the effects of hand and spatial conditions on a visual line bisection task with normal right-handers and proposed a normal range of deviation for this task in middle and advanced age. Twentyfour normal dextrals in their fifties and sixties performed a visual line bisection task using either the left or right hand under three spatial conditions : at the midline and in the left and right hemispaces. Our results revealed that performance was significantly affected by the hand used but not spatial conditions : Left hand performance was significantly further leftward than right hand performance. There was no significant interaction between the hand and spatial conditions. The mean deviation of the right hand was $2.2 \%$ of the half line length to the right of the true center. The possibility of left unilateral spatial neglect should be considered if a patient bisects a line with a deviation greater than $10 \%$ of the half line length to the right._L line bisection; normal range; effect of used hand; effect of hemispace

The visual line bisection task is a common test for detecting unilateral spatial neglect (USN). When required to bisect a horizontal line, patients with left USN are expected to mark the midpoint to the right of the true center, because they neglect the left side of the line.

There have only been a few studies on the performance of a visual line bisection task in normal subjects. Schenkenberg et al. (1980) had 20 hospitalized, non-neurological, right-handed control patients bisect lines and reported that they had a tendency to bisect lines to the left of center when using their left hand, and on or slightly to the right of center when using their right hand. Bradshaw et al. (1985) found that normal students using their right hand demonstrated a significant tendency to bisect to the left of the true center on both a visual line and a tactile rod bisection task. Ferro et al. (1987) studied 14 normal controls and concluded that the subjective midpoint were slightly deviated to the left of the

Received July 12, 1990 ; revision accepted for publication July 25, 1990. 
true midline when they used their right hand. Scarisbrick et al. (1987) had 24 normal college students ( 12 right-handed and 12 left-handed) perform a visual line bisection task with each hand, and found that left-hand perfomances were significantly further leftward than right-hand performances.

One factor important to a better understanding of the USN phenomenon is whether neglect is influenced by the spatial position in which a stimulus is presented. In previous line bisection task studies, the effects of spatial position have been studied with USN patients in the visual modality (Heilman and Valenstein 1979; Riddoch and Humphreys 1983) and normal subjects in the tactile one (Bowers and Heilman 1980; Bradshaw et al. 1983; Hatta and Yamamoto 1986). Heilman and Valenstein (1979) investigated cueing and hemispace effects with USN patients, namely, they cue patients to process the neglected end of lines and varied the spatial condition of stimulus presentation. Only the spatial condition affected performance. Riddoch and Humphreys (1983), on the contrary, reported that the cues affected performance but spatial conditions. In normal subjects, a spatial condition effect has been investigated only with tactile line bisection tasks (Bowers and Heilman 1980 ; Bradshaw et al. 1983 ; Hatta and Yamamoto 1986). Bowers and Heilman (1980) found a hemispace effect while other studies did not (Bradshaw et al. 1983 ; Hatta and Yamamoto 1986). So far no report has been published on the effect of spatial conditions on visual line bisection tasks with normal subjects. In fact, even though the stimuli in some studies (Bradshaw et al. 1983 ; Scarisbrick et al, 1987; Schenkenberg et al. 1980) were presented in three positions on a page; i.e., in left, midline and right position, the effect of spatial conditions has not been taken into account.

The purpose of this study was to investigate the effects of spatial conditions and the hand used on a visual line bisection task in normal subjects having an age comparable to that of cerebrovascular patients in whom neglect phenomenon are studied.

\section{MEthods}

Subjects

Twenty four general medical patients (12 males and 12 females) with no evidence of cerebral disease were examined. They were randomly selected from the inpatients of Iwate National Hospital and Miyagi National Hospital. The subjects were in their fifties or sixties with a mean age of 61.6. All subjects reported that they were right-handed.

\section{Stimuli and procedure}

The stimulus was a black horizontal line $0.5 \mathrm{~mm}$ thick printed in the center of a white piece of paper $18 \mathrm{~cm} \times 25.5 \mathrm{~cm}$ in size. Sixteen different stimuli were constructed for one block, using four different line lengths $(80,120,160,200 \mathrm{~mm})$, with each trial repeated four times. The subjects sat in front of a table and were required to bisect a line presented on the table in just front of them (i.e., middle hemispace). Under the left hemispace condition, the stimuli paper was placed in such a way that the right end was located $15 \mathrm{~cm}$ to the left of the subject's body midline. Under the right hemispace condition, the left end of the 
paper was located $15 \mathrm{~cm}$ to the right of the subject's body midline. Subjects were instructed to mark the midpoint of the line. Each session consisted of three blocks of 16 trials each. Each block was carried out under a different spatial condition (i.e., left hemispace, right hemispace, middle hemispace). Half of the trials under each spatial condition were completed with the right hand and the other half with the left hand. The order of the spatial conditions and the hand used were counterbalanced among subjects. Consequently, all combinations of the hand and spatial position were obtained for each subject.

Analysis

The distance from the left side of the horizontal lines to the subject's mark was measured to within $1 \mathrm{~mm}$ accuracy. This measurement was converted to a percent deviation score (Schenkenberg et al. 1980) using the formula below :

$$
\text { Percent deviation }=\frac{\text { measured left half-true half }}{\text { true half }} \times 100
$$

This formula yields negative values for deviations to the left of center on horizontal lines and positive values for deviations to the right of center. Data were analyzed with a three-way ANOVA with the hand (right, left) and spatial conditions (right, left, midline) as within-subjects factors and sex (male, female) as a between-subjects factor. In addition, single t-tests were performed to determine if the deviations from the midpoint were significantly different from zero.

\section{Results}

An average percent deviation was calculated separately for each hand and each spatial condition. Table 1 shows the average percent deviation among subjects as a function of the hand and spatial conditions.

The results of the analysis of variance revealed a significant hand effect $(\mathrm{F}=$ $14.75, \mathrm{dF}=1,44, p<0.01)$, with left-hand performances being significantly further leftward $(\overline{\mathrm{x}}=-0.12 \%$, S.D. $=3.67)$ than right-hand performances $(\overline{\mathrm{x}}=2.20 \%$, S.D. $=3.92)$. Sex and spatial conditions, however, had no significant effects. All interactions were not significant.

TABLE 1. The mean percent deviation of right and left hand performance on a visual line bisection task under three different spatial conditions : left hemispace, midline, and right hemispacce

\begin{tabular}{cccc}
\hline \multirow{2}{*}{ Space } & \multicolumn{2}{c}{ Hand } & Mean \\
\cline { 2 - 3 } & Left & Right & \\
\hline Left & -1.06 & 2.13 & 0.54 \\
S.D. & & & 3.86 \\
Middle & 0.53 & 2.64 & 1.59 \\
S.D. & & & 3.66 \\
Right & 0.18 & 1.84 & 1.01 \\
S.D. & & & 3.46 \\
Mean & -0.12 & 2.20 & 1.04 \\
S.D. & 3.69 & 3.27 & 3.66 \\
\hline
\end{tabular}


When tested against zero, right-hand performance deviated significantly to the right of center for all spatial conditions (left $=2.13 \%$, midline $=1.59 \%$, right $=$ $1.01 \%, p<0.01)$. Left-handed performances were not significantly different from zero for any conditions $(\mathrm{left}=-1.06 \%$, midline $=0.53 \%$, right $=0.18 \%$ ).

\section{Discussion}

Our data indicate that the performance of visual line bisection is influenced by the hand used.

To date, no reports have investigated the effect of spatial conditions on visual line bisection tasks in normal right-handers. In the tactile modality, Bowers and Heilman (1980) found a significant hemispace effect and an interaction of hand by hemispace, and proposed the hemispace hypothesis to account for these findings. Other tactile studies (Bradshaw et al. 1983; Hatta and Yamamoto 1986) and present results in the visual modality, however, do not support this hypothesis.

The hand effect did influence performance, the leftward displacement being significantly more marked with in the left hand than with the right hand. Similar findings have been reported by Scarisbrick et al. (1987) and Schenkenberg et al. (1980) although the latter authors only presented the mean percent deviation scores for each hand and did not analyze whether the performance of each hand differed significantly. In tactile line bisection tasks, previous reports (Bowers and Heilman 1980; Bradshaw et al. 1983; Hatta and Yamamoto 1986) did not note any effect of the hand used.

Finally, with regard to the subjective midpoint from the true center, our results showed a slight deviation to the right, which was $2.2 \%(0.03 \mathrm{~mm})$ with the right hand and $0.1 \%(1.44 \mathrm{~mm})$ with the left hand. In previous reports, there was a slight deviation to the left with either hand performance. One reason for this discrepancy may be difference in the age of subjects, which in our study was around 60 years and thus to matched the age of cerebrovascular patients, while the subjects of other studies were young college students. The results of the only study carried out in hospital controls (mean age of forty-nine, Shenkenberg et al. 1980) came closest to ours with a slight deviation to the right when compared with the results of young subjects ; i.e., a $2.9 \%$ deviation to the left in the left-hand performance and a $0.9 \%$ one to the right in right hand performance. Clinically, it is essential to delineate the normal variability for the visual bisection task. From our results obtained with normal subjects of middle and advanced age, the $95 \%$ reliant section ( $\overline{\mathrm{x}} \pm 2$ s.D. $)$ is $-5.5 \sim 9.9 \%$ for right-hand performance and -4.3 $\sim 8.7 \%$ for left-hand performance. Therefore, if a patient bisects a line with a deviation of greater than $10 \%$ of half the line length to the right of true center, the possibility of the left USN could be considered. In those cases we should investigate on other tasks, e.g., copy figures and line cancellation, for the confirmation of the left USN. 


\section{References}

1) Bowers, D. \& Heilman, K.M. (1980) Pseudoneglect: Effects of hemispace on a tactile line bisenction task. Neuropsychologia, 18, 491-498.

2) Bradshaw, J.L., Nettleton, N.C., Nathan, G. \& Wilson, L. (1983) Head and body space to left and right, front and rear. Neuropsychologia, 21, 475-486.

3) Bradshaw, J.L., Nettleton, N.C., Nathan, G. \& Wilson, L. (1985) Bisecting rods and lines: Effects of horizontal and vertical posture on left side underestimation by normal subjects. Neuropsychologia, 23, 421-425.

4) Ferro, J.M., Kertesz, A. \& Black, S.E. (1987) Subcortical neglect: Quantitation, anatomy, and recovery. Neurology, 37, 1487-1492.

5) Hatta, T. \& Yamamoto, M. (1986) Hemispheric asymmetries in a tactile bisection task : Effects of hemispace of presentation. Neuropsychologia, 24, 265-269.

6) Heilman, K.M. \& Valenstein, E. (1979) Mechanisms underlying hemispatial neglect. Ann. Neurol., 5, 166-170.

7) Riddoch, M.J. \& Humphreys, G.W. (1983) The effect of cueing on unilateral neglect. Neuropsychologia, 21, 589-599.

8) Scarisbrick, D.J., Tweedy, J.R. \& Kuslansky, G. (1987) Hand preference and performance effects on line bisection. Neuropsychologia, 25, 695-699.

9) Schenkenberg, T., Bradford, D.C. \& Ajax, E.T. (1980) Line bisection and unilateral visual neglect in patients with neurologic impairment. Neurology, 30, 509-517. 